\title{
Anxiety, psychosis and substance use: prevalence, correlates and recognition in an outpatient mental health setting
}

\author{
KM Wyman',JA Chamberlain', DJ Castle ${ }^{2}$ \\ 'Frameworks for Health, St Vincent's Health, Melbourne, Australia \\ 2Department of Psychiatry, St Vincent's Health and The University of Melbourne, Melbourne, Australia
}

\begin{abstract}
Objective: The current study examined anxiety in people with substance use disorder (SUD) and a psychotic disorder. It is hypothesised that: anxiety disorders (AD) would be highly prevalent (greater than 20\%) in people identified as having SUD and psychotic disorders; those with comorbid $\mathrm{AD}$ would fair worse than those without, on measures of quality of life, severity of substance dependence, locus of control, suicidality and psychotic symptoms; and, the presence of such anxiety disorder symptomatology would be under-detected by their mental health case mangers. Method: Outpatients with co-occurring substance use disorder and psychosis were interviewed to collect data about their diagnosis, depressive and psychotic symptoms, severity of dependence on substances, quality of life and locus of control. Participants' case managers made blind ratings of the presence and severity of participants' anxiety. Results: Fifty eight percent of participants had at least one anxiety disorder. Of these, 56\% - 70\% had case managers who had identified them as having an anxiety problem. The occurrence of anxiety disorder was associated with more external locus of control, greater intensity of suicidal thinking, worse psychotic symptoms, lower quality of life and greater severity of dependence on substances. Conclusion: This research suggests anxiety in people with substance use disorder and psychosis is highly prevalent, is associated with poorer outcomes, and is under detected by case managers. Further examination of this issue may lead to important advances in the treatment and prognosis of people with substance use disorder and psychosis.
\end{abstract}

Keywords: Anxiety disorders; Substance-related disorders; Psychotic disorders; Comorbidity; Diagnosis; Prevalence.

Received: 27-05-2010

Accepted: 13-07-2010

doi: http://dx.doi.org/10.4314/ajpsy.v14i3.5

\section{Introduction}

The "dual diagnosis" of psychosis and substance use disorders (SUD) has recently attracted significant clinical and research attention.1,2 Such dual diagnosis is common, with lifetime rates of substance abuse in those with psychotic disorders varying according to setting, ascertainment and also definition of substance use, but a review of the international literature concluded an overall rate for any substance abuse disorder to

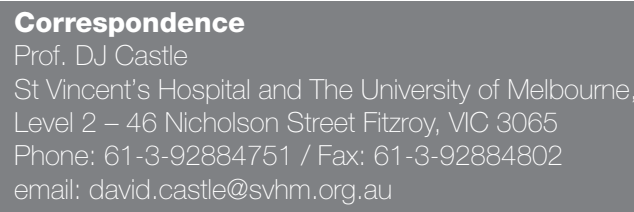

be of the order of 40-60\%. Individuals with schizophrenia and SUD fare worse than their counterparts without SUD in a number of domains including: physical health; self-harm; suicide; engagement, retention and adherence in psychiatric treatment; and psychiatric treatment outcomes. ${ }^{3}$ Fortunately a number of treatment programs to address dual diagnosis have been developed. ${ }^{4-6}$ What is often missed, though, is that people with such dual diagnosis are also at risk of other comorbidities, and these might also impact on both longitudinal outcome and treatment response. ${ }^{7}$ Anxiety disorders (AD) are particularly important in this regard, as they are elevated in both those with schizophrenia and those with SUD, and add further complexity to the clinical picture.

It is well established that in both community and treated samples, AD and SUD commonly co-occur-11; thus, people with 
an $\mathrm{AD}$ are more likely to have a substance use disorder and vice versa. Similarly, there is an elevated rate of $\mathrm{AD}$ in people with schizophrenia. ${ }^{12-16}$

With respect to particular $\mathrm{AD}$, rates of panic disorder in schizophrenia are of the order of 5\% to 35\%.14-19 Obsessivecompulsive disorder (OCD) also commonly co-occurs with schizophrenia. Studies have found $29 \%$ of an inpatient sample ${ }^{17}$ and $37.5 \%$ of an outpatient sample ${ }^{14}$ to have OCD in addition to schizophrenia. The picture is similar for social phobia, with rates of $16.1 \%$ in inpatient ${ }^{17}$ and $37.5 \%$ in outpatient samples ${ }^{14}$ of people with schizophrenia. Other $\mathrm{AD}$ also seem to also co-occur with schizophrenia, but less consistently. ${ }^{14,17}$ Huppert and Smith ${ }^{14}$ found people with schizophrenia and an $\mathrm{AD}$ often have more than one AD diagnosis. In their outpatient sample of people with schizophrenia $28 \%$ had at least one $\mathrm{AD}, 28 \%$ had two $\mathrm{AD}$, and $6.2 \%$ had three or four $\mathrm{AD}$.

Thus it is clear that $\mathrm{AD}$ co-occur with SUD and with schizophrenia. This might lead one to predict that $\mathrm{AD}$ is even more highly prevalent in dual diagnosis populations, i.e. those people with both schizophrenia and SUD. Hitherto, however, few studies have examined correlates between psychotic disorder, anxiety disorders and substance use disorders. In clinical samples, people with schizophrenia and anxiety symptoms ${ }^{18}$ or social phobia ${ }^{20}$ are more likely to have a SUD compared to their non-anxious peers. However, other studies have produced contradictory results. For instance, Labbate et al. ${ }^{21}$ found, in a sample of people with schizophrenia or schizoaffective disorder, that a lifetime history of SUD was not associated with current or past panic attacks or panic disorder. Similarly, another study of outpatients with schizophrenia or schizoaffective disorder compared matched samples of those with and without SUD. Whilst those with SUD more often had a history of trauma, they did not have statistically significant higher rates of PTSD symptoms. Furthermore, there was no difference between those with and without SUD on a measure of trait and state anxiety. ${ }^{22}$ Given that each of these studies have examined different $\mathrm{AD}$ it is difficult to synthesise their results.

Goodwin and colleagues ${ }^{8}$ sampled 184 consecutive inpatients with schizophrenia, 31.5\% of whom had an AD and 35.3\% a history of SUD. After examining the association between discrete $\mathrm{AD}$ and SUD they found panic attacks to be significantly associated with increased likelihood of having a history of SUD. There was no association between a history of SUD and other AD (OCD, specific phobia, social phobia, agoraphobia). A limitation of that study is the exclusion of people with current substance abuse; one would expect this to reduce any observed relationship between specific AD and SUD.

It is also clear that $\mathrm{AD}$ comorbidity is often missed in clinical practice. Shear et al. ${ }^{23}$ found that only $24 \%$ of patients who had a primary $\mathrm{AD}$ identified on the SCID had an $\mathrm{AD}$ diagnosis noted in their outpatient clinic medical record. Similarly, in an outpatient sample of people with schizophrenia only 10\% who were found to have an $\mathrm{AD}$ on the Anxiety Disorders Interview Schedule also had this disorder recorded in their medical record. ${ }^{14}$ And in a separate sample of inpatients with schizophrenia, schizoaffective disorder or bipolar disorder, only 5\% of those identified as having an $\mathrm{AD}$ on the SCID were noted in their medical record as receiving treatment for an $\mathrm{AD} .{ }^{24} \mathrm{AD}$ are more likely to remain undetected in usual clinical practice than psychotic disorders, SUD $^{25}$ and personality disorders. ${ }^{26}$

The implications of AD comorbidity in schizophrenia are substantial. For example in people with schizophrenia, those with panic disorder have higher rates of other mental illnesses, more psychotic symptoms and more suicidality. ${ }^{3}$ Panic disorder in this population may also lead to poor treatment outcomes and excessive service utilisation. ${ }^{13}$ Similarly, anxiety may negatively impact the course and outcome of schizophrenia and impair quality of life. ${ }^{14}$ Anxiety, rather than schizophrenia, might be the source of disability ${ }^{27}$ or may contribute to and maintain the psychotic symptoms..$^{15}$ Thus, the recognition of anxiety in people with schizophrenia is important. More accurate diagnosis may mean that treatment outcome can be better predicted,

comorbidities are more likely to be treated, and patients may be more satisfied. ${ }^{28}$ This in turn may result in an improved working alliance and greater treatment adherence. ${ }^{28}$

This brief review highlights: (1) anxiety is present in both SUD populations and psychotic populations; (2) a limited amount of research suggest a co-occurrence of SUD, AD and psychotic disorders, (3) AD tend to be poorly detected in clinical practice, and (4) $\mathrm{AD}$ are associated with poorer outcomes for people with schizophrenia.

This study explored the prevalence, and clinical correlates of anxiety disorders in a sample of people identified as having a dual diagnosis of psychosis and a SUD. We also examined the rate of detection of these comorbidities by the patients' case managers. Our specific hypotheses were: that:

(1) Anxiety disorders would be highly prevalent (greater than 20\%) in people identified as having SUD and psychotic disorders.

(2) Those with comorbid AD would fair worse than those without, on measures of quality of life, severity of substance dependence, locus of control, suicidality and psychotic symptoms.

(3) The presence of such anxiety disorder symptomatology would be under-detected by their mental health case mangers.

\section{Method}

\section{Sample and recruitment}

The sample was recruited as part of a randomised controlled trial (RCT) of an integrated group based intervention for substance use and psychosis. Participants were from four public area mental health services in metropolitan and rural Victoria, Australia. All participants were outpatients and referred to the study by their case manager. Inclusion and exclusion criteria were a current DSM-IV diagnosis of schizophrenia or an affective psychosis and problematic substance use or dependence; age 16 or above; able to converse in English; and, absence of developmental disability or amnesic syndrome or other cognitive impairment that would impair ability to learn from the intervention. To minimise selection biases and maximise referrals, case managers were asked to keep a low threshold for referral to the study. Education about the study was provided to all case managers and psychiatrists within each service; extra work demands for case managers as a consequence of referral was kept to a minimum; and researchers met with individual case managers and attended clinical reviews in order to identify potential participants and streamline referral to the study. The study was approved by the Human Subjects Review Committees of the participating health services and participants give informed, voluntary and written consent. 
Participants were interviewed prior to randomisation by research clinicians, completing the following measures:

Mini International Neuropsychiatric Interview (MINI) Version 529: a structured diagnostic interview assessing the presence or absence of 17 Diagnostic and Statistical Manual IV (DSM-IV) ${ }^{30}$, Axis I disorders; it has established validity and reliability. ${ }^{31,32}$

Montgomery and Asberg Depression Rating Scale (MADRS) ${ }^{33}$ : a 10-item observer-rated scale. Each item relates to observed or reported depressive symptoms and is rated on a 7-point likert scale. Higher scores indicate more severe depressive symptomatology.

Brief Psychiatric Rating Scale (BPRS) - 16 item version ${ }^{34}$ : an observer rated scale that assesses positive symptoms of psychosis as well as general affective and anxiety symptoms. Higher scores indicate more severe psychopathology. This scale is widely used and has adequate psychometric properties. ${ }^{35}$ In addition to the total score, a number of subscales can be derived from the BPRS. For the purposes of this study we examined total score and three subscale scores: thinking; anxiety / depression; hostility / suspicion.

The Severity of Dependence Scale $(S D S)^{36}$ : The SDS measures the degree of dependence on illicit drugs. It consists of 5 items rating the psychological aspects of dependence on 4-point likert scales. ${ }^{35}$ Higher scores indicate greater dependence. It has good psychometric properties. ${ }^{35}$

Australian World Health Organisation Quality of Life Scale - Brief (WHOQOL-BREF) ${ }^{37}$ : This 26-item self-report scale measures four domains of quality of life, viz.: physical, psychological, social relationships and environmental. Further single items assess overall quality of life and general health.

Locus of Control of Behaviour Scale ${ }^{38}$ : measures perception of personal responsibility for certain behaviours. Higher scores indicate external locus of control whereas lower scores indicate internal locus of control.

Opiate Treatment Index (OTI) drug use section ${ }^{39}$ : a structured interview which obtains information about the amount of alcohol and tobacco, and type and amount of illicit drugs used over three most recent occasions of use for each substance.

Qualified research interviewers, all experienced mental health professionals, conducted interviews according to a research interviewing protocol and procedure manual.

Participants' case managers independently completed Health of the Nation Outcome Scale (HoNOS) Short form. ${ }^{40}$ They were blinded to the current research hypothesis. The HoNOS is a 12-item, observer rated scale routinely used in Victorian Mental Health Services. All case managers are trained in the use of the HoNOS. For the purpose of this analysis we focused on question 8, which asks case managers to indicate whether the client has any "other mental and behavioural problems" (phobias; anxiety and panic; obsessional and compulsive problems; reactions to severely stressful events and traumas; dissociative problems; somatisation; problems with appetite; sleep problems; sexual problems; problems not specified elsewhere). If such a problem is present its severity is rated on a 5-point likert scale. Higher scores indicate greater severity. If more than one problem is present, the most important one is recorded.

\section{Statistical analyses}

The hypothesis that the prevalence of one or more anxiety disorders would be greater than $20 \%$ was tested using a single sample binomial test. Confidence intervals for the prevalence estimates for the anxiety disorders were calculated using exact binomial confidence intervals with a confidence level of 95\%.

Associations between psychometric data, demographics and $\mathrm{AD}$ were investigated using ordinal logistic regression. The number of $\mathrm{AD}(0,1,2$, or $>2)$ for each participant (based on the MINI) was used as the response variable. In the absence of specific information on the severity of each AD identified by the MINI for each participant, this choice of response variable makes better use of the available information than a simple binary variable indicating whether an $\mathrm{AD}$ is present, while avoiding assuming that the number of $\mathrm{AD}$ is on an interval scale. Missing items in the psychometric scales and age were imputed using multiple imputation. ${ }^{41}$ We used the ice Stata command ${ }^{42}$ to generate imputations and estimates were combined using the mim Stata prefix command. ${ }^{43}$

The candidate explanatory variables for a multiple regression were screened with univariate ordinal logistic regression. ${ }^{44}$ Those with a p-value less than 0.2 and not measuring anxiety were evaluated further using correlation analysis (Pearson and Spearman) followed by a multiple ordinal logistic regression with explanatory variables chosen on clinical and statistical grounds.

Recognition of anxiety by case managers was analysed using the kappa statistic ${ }^{45}$ to determine the agreement between the MINI diagnosis and case manager report on the HoNOS. In this study, each participant's $\mathrm{AD}$ profile was rated once by a clinician using the MINI and once by their case manager using the HoNOS item 8. Consequently, it was not possible to separate the variance due to the measurement method (MINI or HoNOS) and the variance due to the rater performing the particular measurement. It is only possible to calculate a measure of the overall agreement between the two measurement processes: MINI diagnosis by a clinician versus HoNOS diagnosis by a case manager.

When a non-anxiety problem was specified as the main problem in item 8 of the HoNOS there was ambiguity as to whether the case manager recognised an anxiety problem or not. This issue was dealt with by considering three separate cases in our analysis (see Results).

Based on a one-tailed single sample binomial test with prevalence of $\mathrm{AD}$ of $20 \%$ as the null hypothesis and a prevalence of $50 \%$ as the prevalence that it is important to be able to detect, the sample size required for greater than $99 \%$ power with a significance level of 0.05 is 42 participants. ${ }^{46}$ With a sample of 87 available from the RCT, this study is well powered to examine prevalence of AD.Stata 9.2 under Windows XP was used for the analysis.

\section{Results}

Sample characteristics

The total number of participants randomised in the RCT was 102. Of these, 87 participants had sufficient baseline data to analyse. The sample selection procedure was determined by what data was available, so that if there was any bias it would be 
most likely due to participants selectively not responding rather than research clinicians choosing the sample. The age range of the 87 participants was 19 to 50 years and $22 \%$ were female. Primary diagnoses on referral were: schizophrenia (any type) 56\% (49/87); schizoaffective disorder 23\% (20/87); bipolar (and not schizoaffective) $2 \%$ (2/87) and other psychotic disorders including schizophreniform psychosis 18\% (16/87). The total numbers for the MINI and OTI differ as some baseline assessments contained missing data. Sixty percent (50/84) had current alcohol abuse or dependence according to the MINI, and 75\% (63/84) had current substance abuse or dependence. Based on the OTI, the prevalence of drugs used in the previous 28 days was: alcohol 80\% (66/82), cannabis 57\% (47/83), amphetamines 12\% (10/83), cocaine 0\% (0/82), hallucinogens 5\% (4/81), inhalants 0\% (0/82), heroin 5\% (4/83), tobacco 96\% (78/81). The length of time within mental health services ranged from 1 to 32 years (mean 9.2 years; standard deviation (SD) 8.1 years). Employment status was reported for 73 participants. Of these, 78\% (57/73) were unemployed, 19\% (14/73) had casual or part-time employment and 3\% (2/73) were employed full-time. Prescribed psychotropic medications were recorded for 79 participants. Ninety-four percent (74/79) were prescribed antipsychotics, 24\% (19/79) mood stabilisers, 20\% (16/79) anti-depressants, 8\% (6/79) anxiolytics, and 4\% (3/79) hypnotics Forty-two percent (33/79) of participants were prescribed more than one class of psychotropic medication.

\section{Prevalence of $A D$}

Data from the MINI showed that the prevalence of at least one $A D$ was significantly greater than $20 \%(\mathrm{p}<0.001)$. The prevalence estimate was $58.6 \%$ with $95 \%$ confidence interval $47.6 \%$ to $69.1 \%$. In our sample, $35.6 \%$ of the participants met criteria for one $\mathrm{AD}, 12.6 \%$ had two $\mathrm{AD}$, and $10.4 \%$ had three or more $\mathrm{AD}$. The prevalence of different types of $\mathrm{AD}$ were: generalised anxiety disorder $25.3 \%$ (CI 16.6\% to $35.7 \%$ ), agoraphobia $21.8 \%$ (CI 13.7\% to 32.0\%), social phobia 19.5\% (CI $11.8 \%$ to $29.4 \%$ ), panic disorder $14.9 \%$ (CI $8.2 \%$ to $24.2 \%$ ), post traumatic stress disorder $10.3 \%$ (CI 4.8\% to 18.7\%) and obsessive compulsive disorder $4.6 \%$ (CI $1.2 \%$ to $11.4 \%$ ).
Association between observed $A D$ and demographic and psychometric data

The results for the univariate screening of 13 explanatory variables are shown in Table I. Gender, age and WHO-QOL BREF satisfaction with health did not reach statistical significance $(p>0.2)$ in their association with the observed $A D$ and were dropped from further analysis. The significant results for MADRS total score, BPRS total score and BPRS anxiety / depression were not surprising because these scales contain items measuring anxiety directly and we expected them to be associated with the diagnosis of an $\mathrm{AD}$. Consequently, these three variables were also dropped from further analysis, leaving seven potential explanatory variables: SDS, LCB, BPRS thinking, BPRS hostility /suspicion, WHOQOL-BREF quality of life, WHOQOL-BREF psychological health and MADRS suicide item.

The correlation analysis of these remaining variables is shown in Fig 1. We used this correlation analysis to choose three explanatory variables to include in the final multiple ordinal logistic regression; SDS total; combined BPRS thinking and hostility/suspicion sub-scales (herein referred to as BPRS thinking/hostility/suspicion); and WHOQOL-BREF quality of life. These variables were chosen as representative variables because, among the correlates, they were the most conceptually distinct from each other and were not strongly correlated (Figure 1). The response variable for the regression was the number of $\mathrm{AD}$.

The results of the multiple ordinal logistic regression are shown in Table II. For a given combination of explanatory variables, the model predicts probabilities of the number of $\mathrm{AD}$, as illustrated in Fig. 2. The confidence regions (not shown in Figure 2 for clarity) were wide: for example, with explanatory variables at their means, the probability of one or more $\mathrm{AD}$ had a 95\% CI with width 0.3. Consequently, the model predictions are not precise but they do indicate a pattern of association between increasing numbers of $\mathrm{AD}$ and worse ratings on the SDS, WHOQOL-BREF quality of life item and BPRS thinking/hostility/suspicion.

In the case of SDS, as the total score increases, the probability of being free of any $\mathrm{AD}$ decreases, and the probability of having 1 or more, 2 or more and 3 or more $A D$

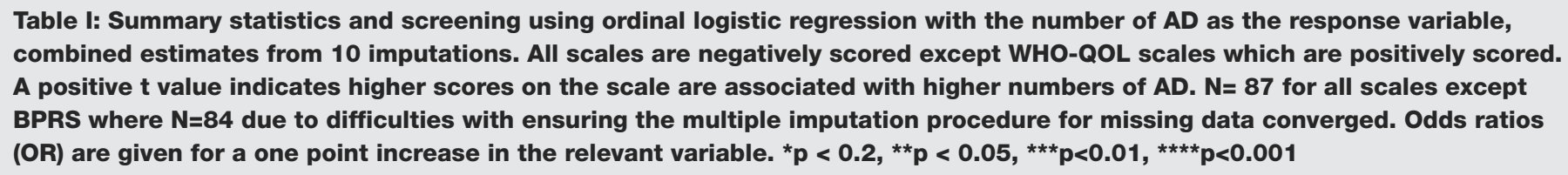

\begin{tabular}{|c|c|c|c|c|c|c|}
\hline Variable & Mean & $S D$ & t & $O R$ & $95 \% \mathrm{Cl}$ & $p$ \\
\hline Gender & $1.22(M=1, F=2)$ & 0.42 & -0.34 & 0.84 & 0.29 to 2.38 & 0.7 \\
\hline Age (yrs) & 31.58 & 8.63 & 0.13 & 1.00 & 0.96 to 1.05 & 0.9 \\
\hline SDS total & 7.83 & 3.88 & 1.83 & $1.15^{\star}$ & 0.99 to 1.35 & 0.07 \\
\hline LCB total & 35.19 & 10.91 & 2.42 & $1.05^{\star \star}$ & 1.01 to 1.10 & 0.02 \\
\hline BPRS total & 1.68 & 0.53 & 4.45 & $6.67^{\star \star \star \star}$ & 2.89 to 15.38 & $<0.001$ \\
\hline BPRS thinking & 1.58 & 0.84 & 2.93 & $1.70^{\star \star \star}$ & 1.19 to 2.43 & 0.003 \\
\hline BPRS anxiety/depression & 2.11 & 0.81 & 3.96 & $3.47^{\star \star \star \star}$ & 1.87 to 6.44 & $<0.001$ \\
\hline BPRS hostility/suspicion & 1.46 & 0.62 & 2.49 & $2.52^{\star \star}$ & 1.21 to 5.23 & 0.01 \\
\hline WHOQOL-BREF quality of life & 3.15 & 1.08 & -3.21 & $0.52^{\star \star \star}$ & 0.35 to 0.78 & 0.001 \\
\hline WHOQOL-BREF satisfaction with health & 2.93 & 1.06 & -0.79 & 0.83 & 0.53 to 1.31 & 0.4 \\
\hline WHOQOL-BREF psychological health & 51.70 & 17.00 & -2.67 & $0.96^{\star \star \star}$ & 0.93 to 0.99 & 0.008 \\
\hline MADRS total & 10.86 & 9.58 & 3.47 & $1.11^{\star \star \star}$ & 1.05 to 1.18 & 0.001 \\
\hline MADRS suicide item & 0.72 & 1.21 & 2.25 & $1.66^{\star \star}$ & 1.07 to 2.60 & 0.03 \\
\hline
\end{tabular}


Figure 1: Significant correlations between candidate explanatory variables, after screening to remove weak ( $p>=0.2$ ) predictors of $A D$ and those measuring anxiety directly, $r=$ Pearson product moment correlation, $\rho=$ Spearman rank correlation. The Sidak adjustment for multiple tests was used with significance level 0.05

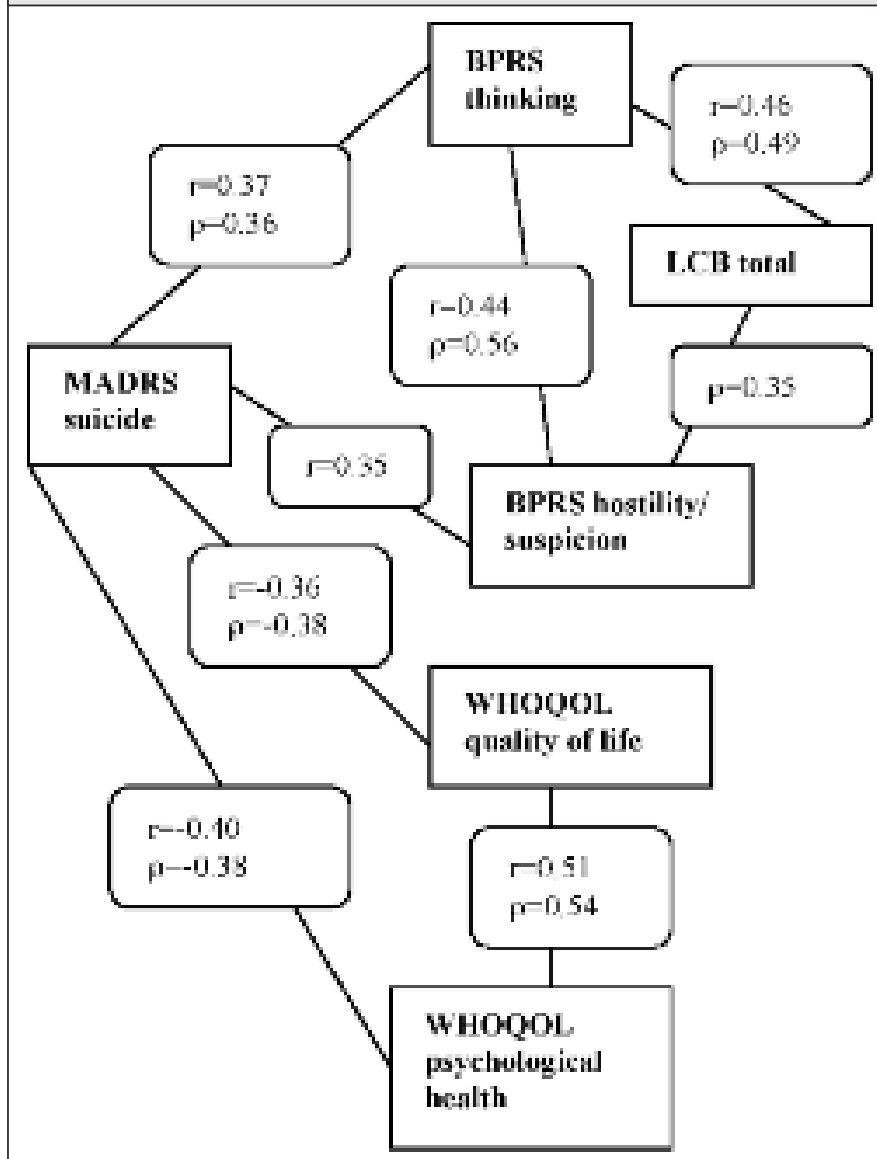

SDS total

increases. For example an SDS score of 14 indicates a probability of approximately $20 \%$ for having 3 or more AD. Similar patterns were seen with the WHOQOL-BREF quality of life item (although this scale is positively scored) and combined BPRS thinking/hostility/suspicion.

Thus, the profile of someone at most risk of having an $\mathrm{AD}$ is an individual who scores high on the SDS and BPRS

thinking/hostility/suspicion scores and has a low WHOQOL-BREF quality of life item score. For example scores of 12 on SDS, 2 on WHOQOL and 2.5 BPRS thinking/hostility/suspicion give a 43\% probability of having more than 2 anxiety disorders. In contrast, the profile of someone at least risk of AD is low SDS and BPRS thinking/hostility/suspicion scores and high WHOQOL-BREF quality of life item score. For example, scores of 4 on SDS, 4 on WHOQOL-BREF quality of life and 1 on BPRS

thinking/hostility/suspicion give a 74\% probability of no $\mathrm{AD}$.

\section{Recognition}

Rates of recognition of $\mathrm{AD}$ by their case managers are shown in Table III, where the MINI diagnosis is tabulated against item 8 of the HoNOS. Item 8 of the HoNOS was rated as (a) no problem (b) an anxiety problem (i.e. phobias; anxiety and panic; obsessional
Figure 2: Predicted probabilities of having a particular number of $A D$ as a function of SDS total. Based on the ordinal logistic regression model with the WHOQOL-BREF quality of life item constrained to be 3 and combined BPRS thinking/hostility/suspicion constrained to be 1.52. The response variable for the regression was the number of $A D$

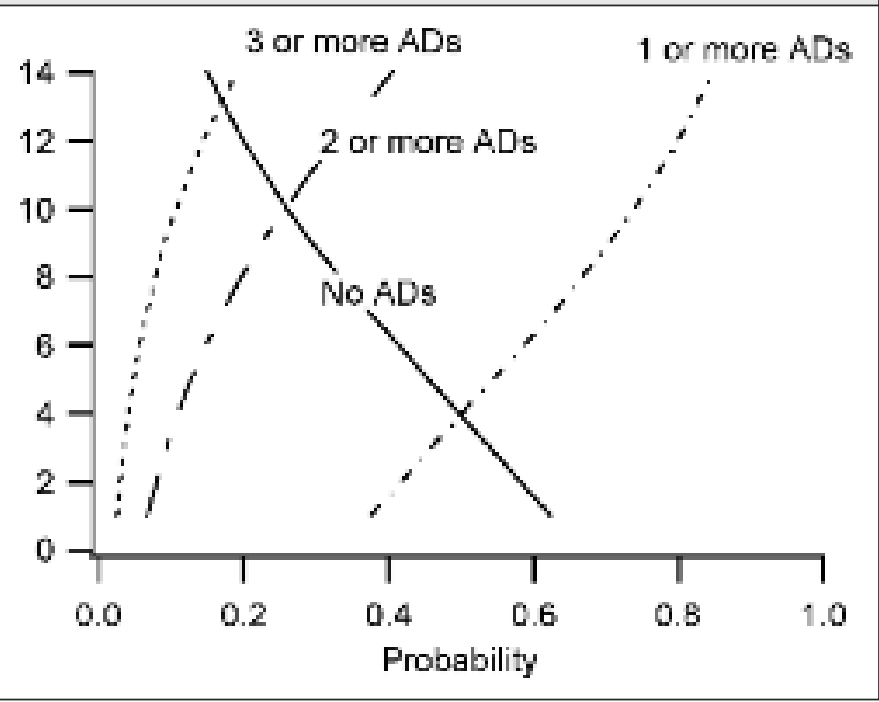

Table II: Multiple ordinal logistic regression using multiple imputation for missing data (10 imputed datasets with $\mathbf{N}=84$ ). The response variable is the number of $\operatorname{AD}(0,1,2$, or $>2)$. Odds ratio (OR) is for a one point increase in the explanatory variable. Cut points are used to link the latent variable to the observed categories

\begin{tabular}{|l|l|l|l|l|}
\hline Explanatory variables: & OR & $t$ & $95 \% \mathrm{Cl}$ & $p$-value \\
\hline SDS total & 1.19 & 2.27 & 1.02 to 1.38 & 0.03 \\
\hline $\begin{array}{l}\text { BPRS thinking/ } \\
\text { hostility/suspicion }\end{array}$ & 2.49 & 2.76 & 1.30 to 4.78 & 0.006 \\
\hline $\begin{array}{l}\text { WHOQOL-BREF } \\
\text { quality of life item }\end{array}$ & 0.55 & -2.68 & 0.35 to 0.85 & 0.008 \\
\hline Cut points: & 1.28 & & $\begin{array}{l}0.12 \text { to } 14.02 \\
0.81 \text { to } 136.00 \\
2.25 \text { to } 409.59\end{array}$ & \\
\hline $\begin{array}{l}\text { Cut 1 } \\
\text { Cut 2 } \\
\text { Cut 3 }\end{array}$ & 30.51 & & & \\
\hline
\end{tabular}

Wald $\chi^{2}(3)=25.55$, Prob $>\chi^{2}<0.0001$, Pseudo $R^{2}=0.12$

Table III: Association between MINI anxiety and HoNOS anxiety

\begin{tabular}{|l|l|l|l|}
\hline HoNOS & $\begin{array}{l}\text { MINI } \\
\text { No Anxiety }\end{array}$ & Anxiety & Total \\
\hline No problem & 10 & 10 & 20 \\
Anxiety problem & 8 & 18 & 26 \\
Non - anxiety problem & 9 & 6 & 15 \\
Invalid/missing & 2 & 6 & 8 \\
\hline Total & 29 & 40 & 69 \\
\hline
\end{tabular}


and compulsive problems) or (c) non-anxiety problems (i.e. reactions to severely stressful events and traumas; dissociative problems; somatisation; problems with appetite; sleep problems; sexual problems; problems not specified elsewhere). There was ambiguity when a case manager specified a non-anxiety problem: the case manager could have recognised an anxiety problem but specified a non-anxiety problem which they thought was more severe than the anxiety problem, or they could have not recognised an anxiety problem, or no anxiety problem was present. This ambiguity was dealt with by considering the following three cases in the analysis when a non-anxiety problem was specified at item 8: (i) case managers recognised the client as having an anxiety problem (ii) case managers did not recognise an anxiety problem and (iii) case managers recognised the anxiety problem in agreement with the MINI.

For case (i) the expected agreement assuming random rating was $52 \%$ and the observed agreement was 56\% with $\kappa=0.08$ (slight agreement, standard error $=0.12, z=0.63, p=0.26$ ); for case (ii), the expected agreement was $49 \%$ and the observed agreement was $61 \%$ with $\kappa=0.23$ (fair agreement, standard error $=0.12, \mathrm{z}=1.83, \mathrm{p}=0.03$ ); and for case (iii), expected agreement was 50\% and the observed agreement was 70\% with $\kappa=0.41$ (moderate agreement, standard error $=0.13, z=3.18, p<0.001$ ). This last analysis is the most generous to the case managers HoNOS ratings in terms of assuming their accuracy.

\section{Discussion}

The study reported here explored the prevalence and associations between $\mathrm{AD}$, substance use and psychotic symptoms in a group of patients with a comorbid psychotic illness and SUD. The results demonstrate that $58 \%$ of a sample of people with substance use and psychosis met criteria for at least one anxiety disorder. The presence and number of $\mathrm{AD}$ was associated with more externally (rather than internally) located locus of control, greater intensity of suicidal thinking, worse psychotic symptoms, lower of quality of life and greater severity of dependence on substances. These three latter domains, analysed together, were all strongly associated with the number of $\mathrm{AD}$.

Analysis of case manager's recognition of anxiety problems in this sample suggests the presence or absence of anxiety problems was correctly recognised in clients between 56\% and $70 \%$ of the time. It needs to be emphasised that case managers were only asked whether they observed anxiety problems, not anxiety disorders. Thus the criteria for case manager recognition of anxiety was low, should this criteria have been higher (i.e. anxiety disorder) one can assume that the case managers may have recognised fewer $\mathrm{AD}$. One explanation for case managers not recognising anxiety problems is the tendency to think of psychiatric illnesses as on a hierarchy, with $\mathrm{AD}$ being lower down that hierarchy than psychotic illnesses. Bermanzohn ${ }^{27}$ suggests that hierarchical systems of diagnosis have played a role in the lack of attention given to comorbid diagnoses in schizophrenia as it lead to diagnostic reductionism; "the tendency to reduce all of the symptoms and signs shown by a person with schizophrenia to the schizophrenia alone." (p3). ${ }^{27}$ It should also be pointed out that anxiety-like symptoms can also be consequent upon, or misinterpreted as, substance withdrawal, akathisia, or as part of the manifestation of psychotic symptoms per se.

This study has a number of strengths, including the fact that all subjects met criteria for both a psychotic illness and a SUD. There were few exclusion criteria and the inclusion of people with current substance use is an improvement from previous research. ${ }^{8,21}$ Also, all were assessed using standardised measures with raters 'blind' to the hypotheses of the study. The number of subjects, whilst modest, was sufficient to power the analyses performed. However, the numbers were not sufficient for more complex analyses using other variables, for example service utilisation data. Nor did we include data on treatment response, which is a gap because understanding the implications of comorbidity on treatment response could enhance our management of these patients.

Several limitations of this study are important to note. The variance for the two measurement processes could not be separated out into components due to the measurement method (MINI or HoNOS) and a component for the rater (clinician or case manager). An inter rater reliability study with more than one rating per participant/ method combination (outside the scope of this article) would be required to investigate this. Furthermore, the sample was selected on the basis of referral to a RCT for psychosocial treatment for psychosis and SUD. Although efforts were made to obtain the broadest possible sample, it remains the case those individuals who were not considered by case managers as appropriate for the specified treatment would be selected out, limiting the generalisability of results.

\section{Conclusion}

Our findings do reinforce the high rates of anxiety disorder comorbidity in those with a psychotic illness and substance abuse, and show that anxiety problems are missed in routine clinical practice even by case managers who 'know' their patients well. The results also suggest that, compared to their non-AD peer those individuals with SUD, psychosis and AD fair worse on a number of domains. This line of research may be advanced through conceptualising anxiety on a continuum rather than discrete disorders; investigating the impact of anxiety on treatment outcomes for those with dual diagnosis; and examining the relationship between anxiety, specific SUD and psychosis in larger, epidemiological studies.

\section{Acknowledgements}

This research was supported by NHMRC Project Grant number 236100. We would like to acknowledge the hard work of many people at Frameworks for Health and from Victorian Area Mental Health Services who generously contributed to the Managing Mental Health and Substance Use Project.

\section{References}

1. Cantor-Graae E, Nordstrom LG, MCNeil TF. Substance abuse in schizohrenia: a review of the literature and study of correlates in Sweden. Schizophrenia Research 2001;48:69-82

2. Bellack AS, Bennett ME, Gearon JS. Behavioural treatment for substance abuse in people with serious and persistent mental illness: an handbook for mental health professionals. New York: Routledge; 2007.

3. Kavanagh DJ, Mueser KT, Baker A. Management of comorbidity. In: Teesson M, Proudfoot $H$, editors. Comorbid mental disorders: epidemiology, prevention and treatment. Canberra: Australian Government; 2003. p. 78-120.

4. Minkoff K, Cline CA. Developing welcoming systems for individuals with co-occurring disorders: The role of the comprehensive continuous integrated system of care model. Journal of Dual Diagnosis 2004;1 (1):65-89.

5. Mueser KT, Noordsky DL, Drake RE, Fox L. Integrated Treatment for 
dual disorders: a guide to effective practice. New York: The Guilford Press; 2003.

6. James W, Preston N, Koh G, Spencer C, Kisely S, Castle D. A group intervention which assists patients with dual diagnosis reduce their drug use: a randomized controlled trial. Psychological Medicine 2004:34:983-990

7. Weiss RD, Mirin SM, Frances RJ. The myth of the typical dual diagnosis client. Hospital and Community Psychiatry 1992;43:107-108

8. Goodwin RD, Amador XF, Malaspina D, Yale SA, Goetz RR, Gorman JM. Anxiety and substance use comorbidity among inpatients with schizophrenia. Schizophrenia Research 2003;61:89-95.

9. Grant B, Stinson F, Dawson D, Chou P, Dufour M. Prevalence and cooccurrence of substance use disorders and independent mood and anxiety disorders. Achieves of General Psychiatry 2004;61:807-816.

10. Kushner M, Sher K. The relation between alcohol problems and the anxiety disorders. American Journal of Psychiatry 1990; 147:685-695.

11. Regier D, Rae D, Narrow W, Kaelber C, Schatzberg A. Prevalence of anxiety disorders and their comorbidity with mood and addictive disorders. British Journal of Psychiatry 1998;173 (suppl 34):24-28.

12. Bermanzohn PC, Porto L, Arlow P, Pollack S, Stronger R, Siris S. At issue: hierachical diagnosis in chronic schizophrenia: a clinical study of COoccurring syndromes. Schizophrenia Bulletin 2000;26(3):517-525.

13. Goodwin RD, Lyons JS, J MR. Panic attacks in schizophrenia. Schizophrenia Research 2002;58:213-220.

14. Huppert J, Smith T. Anxiety and schizophrenia: the interaction of subtypes of anxiety and psychotic symptoms. CNS Spectrum 2005; 10(9):721-731.

15. Turnbull G, Bebbington P. Anxiety and the schizophrenic process: clinical and epidemiological evidence. Social Psychiatry and Psychiatric Epidemiology 2001;36:235-243.

16. Pokos V, Castle DJ. Prevalence of comorbid anxiety disorders in schizophrenia spectrum disorders: A literature review. Current Psychiatry Reviews 2006;2:285-307.

17. Cassano G, Pini S, Saettoni M, Rucci P, Dell'Osso L. Occurrence and clinical correlates of psychiatric comorbidity in patients with psychotic disorders. Journal of Clinical Psychiatry 1998;59(2):60 - 68.

18. Moorey H, Soni SD. Anxiety symptoms in stable chronic schizophrenics. Journal of Mental Health 1994;3(2):257-262.

19. Pitch RJ, Bermanzohn PC, Siris S. Panic symptoms in patients with schizophrenia. In: Hwang MY, Bernmanzohn PC, editors. Schizophrenia and comorbid conditions: diagnosis and treatment. Washington: American Psychiatric Press; 2001. p. 79-94.

20. Pallanti S, Querciolo 1, Hollander E. Social anxiety in outpatients with schizophrenia: a relavant cause of disability. The American Journal of Psychiatry 2004;161(1):53-58.

21. Labbate L, Young C, P, Arana GW. Panic disorder in schizophrenia. Canadian Journal of Psychiatry 1999;44(5):488-491

22. Sheller-Gilkey G, Thomas SM, Woolwine BJ, Miller AH. Increasing early life stress and depressive symptoms in patients with comorbid substance abuse and schizophrenia. Schizophrenia Bulletin 2002;28(2):223-231

23. Shear KM, Greeno C, Kang J, Ludewig D, Frank E, Swartz H, et al. Diagnosis of nonpsychotic patients in community clinics. The American Journal of Psychiatry 2000;157(4):581-587.

24. Cosoff SJ, Hafner RJ. The prevalence of comorbid anxiety in schizophrenia, schizoaffective disorder and bipolar disorder. Australian and New Zealand Journal of Psychiatry 1998;32:67-72.

25. Brady S, Rierdan J, Penk W, Losardo M, Meschede T. Post-traumatic stress disorder in adults with serious mental illness and substance abuse. Journal of Trauma and Dissociation 2003;4(4):77-90.

26. Kranzler HR, Ronald RM, Burleson JA, Babor TF, Apter A, Rounsaville BJ.
Validity of psychiatric diagnosis in patients with substance use disorders: is the interview more important than the interviewer? Comprehensive Psychiatry 1995;36:278-288.

27. Bermanzohn PC, Porto L, Siris S, Stronger R, Hwang MY, Pollack S. Hierarchy, reductionism and comorbidity in the diagnosis of schizophrenia. In: Hwang MY, Bermanzohn PC, editors. Schizophrenia and comorbid conditions: diagnosis and treatment. 1st ed. Washington: American Psychiatric Press; 2001. p. 1-30

28. Zimmerman M. What should the standard of care for psychiatric disanostic evaluations be? Journal of Nervous and Mental Disease 2003;191 (5):281-286

29. Sheehan DV, Y L, Harnett-Sheehan K, Amorim P, Janavs J, Weiller E, et al. The Mini International Neuropsychiatric Interview (M.I.N.I.): The developments and validation of a structured diagnostic Interview. Journal of Clinical Psychiatry 1998;59(suppl 20):22-33.

30. Association AP. Diagnostic and Statistical Manual of Mental Disorders. 4th ed. Washington DC: American Psychiatric Association; 1994.

31. Sheehan DV, Lecruber Y, Harnett-Sheehan K, Janavs J, Weiller E, Keskiner $A$, et al. The validity of the Mini International Neuropsychiatric Interview (M.I.N.I) according to the SCID-P and its relaibility. European Psychiatry 1997;12:224-231

32. Lecrubier Y, Sheehan DV, Weiller E, Amorim P, Bonora I, Harnett-Sheehan $K$, et al. The Mini International Neuropsychiatric Interview (M.I.N.I.). A short diagnostic structured interview: relaibility and vlidity according to the CIDI. European Psychiatry 1997;12:224-231.

33. Montgomery SA, Asberg M. A new depression scale designed to be sensitive to change. British Journal of Psychiatry 1979;134:382-389.

34. Overall JE, Gorham DR. The brief psychiatric rating scale. Psychological Reports 1962;10:799-812.

35. Dawe S, Loxton NJ, Hides L, Kavanagh DJ, Mattick RP. Review of diagnostic screening instruments for alcohol and other drug use and other psychiatric disorders. 2nd ed: Publications Production Unit, Commonwealth Department of Health and Aging; 2002.

36. Gossip M, Darke S, Griffiths P, Hanso J, Powis B, Hall W, et al. The severity of dependence scales (SDS): PSychometric properties of the SDS in English and Australian samples of heroin, cocaine and amphetimine users. Addiction 1995;90:607-614.

37. Murphy B, Herrman H, Hawthorne G, Pinzone T, Evert H. Australian WHOQOL instruments: User's manual and interpretation guide. Melbourne, Australia: Australian WHOQOL Field Study Centre; 2000.

38. Craig AR, Franklin JA, Andrews G. A scale to measure locus of control of behaviour. British Journal of Medical Psychology 1984;57:173-180.

39. Darke S, Ward J, Wall W, Heather N, Wodak A. The Opiate Treatment Index (OTI) Manual. Sydney, Australia: National Drug and Alcohol Research Centre; 1991.

40. Wing J, Curtis R, Beevor A. Health of the nation outcome scales. Trainers guide. London: Royal College of Psychiatrists; 1996.

41. Rubin DB. Multiple Imputation for Nonresponse in Surveys,. New York: Wiley; 2004

42. Royston P. Multiple imputation of missing values: further update of ice, with an emphasis on intercal censoring. The Stata Journal 2007;7(4):445-464.

43. Carlin JB, Galati JC, Royston P. A new framework for managing and analyzing multiply imputed data in Stata. The Stata Journal 2008;8(1):49-67.

44. Long JS, Freese J. Regression Models for Categorical Dependent Variables using Stata. College Station: Stata Press; 2003

45. Fleiss JL, Levin B, Paik MC. Statistical Methods for Rates and Proportions. 3rd ed. New York: Wiley; 2003

46. Kraemer HC, Thieman SH. How many subjects? Statistical Power Analysis in Research,. Newbury Park: Sage; 1987. 